\title{
Whole-genome Resequencing Provides Insights into the Population Structure and Domestication Signatures of Ducks in Eastern China
}

\section{Peishi Feng}

Zhejiang University of Technology https://orcid.org/0000-0001-7981-4514

\section{Tao Zeng}

Zhejiang Academy of Agricultural Sciences

\section{Yang Hua}

Zhejiang Academy of Agricultural Science

\section{Guohong Chen}

Yangzhou University Animal Science and Technology College

\section{Jinping Du}

Hubei Academy of Agricultural Science

\section{Li Chen}

Zhejiang Academy of Agricultural Sciences

\section{Junda Shen}

ZheJiang Academy of Agricultural Sciences

\section{Zhongrong Tao}

Zhejiang Academy of Agricultural Sciences

Ping Wang ( $\nabla$ wangping45@zjut.edu.cn)

Zhejiang University of Technology, Hangzhou https://orcid.org/0000-0002-6716-4451

\section{Lin Yang}

South China Agricultural University College of Animal Science

Lizhi Lu

zhejiang academy of agricultural sciences

\section{Research article}

Keywords: duck, domestication, insulin signaling pathway, population history, artificial selection, adaptation

Posted Date: September 15th, 2020

DOl: https://doi.org/10.21203/rs.3.rs-48906/v1 
License: (c) (i) This work is licensed under a Creative Commons Attribution 4.0 International License. Read Full License 


\section{Abstract}

Background: Ducks are an ancient domesticated animal with high economic value, uesd for its meat, eggs, and feathers. However, the origin of indigenous Chines ducks remains elusive. To address this question, we performed whole-genome resequencing to explore the genetic relationships among the domestic ducks and wild ducks in eastern China and to identify the genomic differences shaped by artificial selections.

Results: The whole genomes of 60 ducks from eastern China were sequenced, with an average effective sequencing depth of $\sim 6 \times$ per individual. The results of population and demographic analysis indicate that all individuals from the same breed gather together, and domestic ducks form a cluster separating from wild ducks. By applying selective sweep analysis, we identified that several candidate genes, important pathways and GO categories associated with artificial selection were functionally related to cellular adhesion, type 2 diabetes, lipid metabolism, the cell cycle, liver cell proliferation, and muscle functioning in domestic ducks.

Conclusion: Genetic structure analysis showed that the domesticated ducks were not direct descendants of the mallard or Spot-billed duck and probably originated from an unknown population. During the long history of artificial selection, domesticated ducks have developed a complex biological adaptation to captivity.

\section{Background}

Domestication is the process of animal adaptation to humans and the captive environment by genetic changes acrosss many generations [1]. Evolutionary biology is centered on the processes of variation, heredity, and selection driven by domestication. The phenotypic and genetic variation among breeds in many species may be influenced by domestication or captivity, such as in pig [2], chickens [3], dogs [4] and yaks [5].

Chinese domestic ducks are among the earliest domesticated fowl in the world dating back to 3000 years before present (YBP) [6]. China is famous for its abundance of waterfowl breeds, there are 31 recognized domestic duck breeds. However, the diversity and origin of Chinese domestic ducks are still debated. Some suggest that Chinese domestic ducks originated from wild mallards (Anas platyrhnchos) [7, 39], while others argue for a hybrid originated wild mallards and spot-billed duck (Anas poecilorhyncha) [8].

Domestic ducks differ from their wild ancestors in morphology $[9,10]$ and behavior $[11]$ because they are bred for egg production and meat production. The economic importance of the domestic ducks has driven research to decode the duck genome. Thus it is necessary to pinpoint the genetic basis of domesticated ducks.

In this study, we sequenced the genomes of 60 individuals from two wild populations, the spot-billed ducks (Anas poecilorhyncha) and mallards (Anas platyrhnchos), and four indigenous Chinese breeds 
(Fenghua (FH), Shaoxing (SX), Shanma (SM), and Cherry Valley Pekin (CV) ducks). The Fenghua duck is used for egg or meat production. Shaoxing and Shanma ducks are Chinese dominant layer breeds, characterized by small bodies size, early maturity and high productivity. The Cherry Valley Pekin duck is famous for its fast growth, high lean rate and high feed conversion ratio. We used whole-genome resequencing approach and transcriptome sequencing methods to explore the genetic relationships among wild and domestic ducks and identified the genomic footprints of selection during the domestication of native ducks.

\section{Results}

We selected 60 individuals from six breeds (spot-billed duck, mallard duck,Fenghua duck, Shaoxing Duck, Shanma Duck and Cherry Valley Pekin Duck) (Fig. 1A). Using the Illumina Genome Analyzer platform, we generated a total of $397.88 \mathrm{~GB}$ of clean data with an average of $6.63 \mathrm{~GB}$ per individuals (Supplementary Table S1). These reads mapped to $95.09 \%$ of the reference genome assembly with 6.52 -fold average depth (Supplementary Table S2). We called 2,809,077 high-quality single nucleotide polymorphic sites (SNPs) for 60 ducks (Supplementary Table S3), 63.92\% (1.8 million) of the high-quality SNPs were located in the intergenic regions, and only $1.94 \%$ ( 0.55 million) were located in the exonic regions (Supplementary Table S4). We identified 42,463 synonymous SNPs and 12,084 nonsynonymous of exons, for a nonsynonymous/synonymous ratio of 0.28 . There were 838,413 SNPs shared between the breeds (Supplementary Fig. S1).

\section{Population Genetic Structure}

To explore relatedness among the domestic ducks, we conducted a principal component analysis (PCA) based on genome wide SNP data. The laying duck breeds (Shaoxing and Shanma ducks) and meat duck breeds (Cherry Valley Pekin duck) were separated by different clusters that were also distinct from the wild populations (spot-billed duck and mallard) and Fenghua duck (Fig. 1A, supplementary Fig. S2). The neighbor-joining $(\mathrm{NJ})$ tree revealed that the individuals from Chinese indigenous breeds were clustered into a subclade, suggesting they have a closer genetic relationgship and potentially derive from a common ancestor (Fig. 1B). To estimate different ancestral proportions, we further performed a population structure analysis with FRAPPE by assuming $K$ ancestral populations (Fig. 1C). When $K=2$, a clear division was observer between the wild ducks and domesticated breeds with slight shared ancestry sequences between these two groups. Moreover, we observed $59 \%$ wild population ancestry of in Fenghua duck. When $K=5$, there was a division between each group except Shaoxing and Shanma ducks.

To examine the population structure with admixture, we inferred the duck population tree for ducks using TreeMix (Fig. 2). One of the three included migration events showed strong gene flow from Fenghua duck into mallard, and the two other migration events estimates a weaker gene flow from Shaoxing duck into spot-billed duck and Shanma duck into Cherry Valley Pekin duck. 


\section{Patterns of Genomic Variation and Linkage Disequilibrium}

The genome-wide average genomic diversity $\left(\theta_{\pi}\right)$ values were $5.949 \times 10^{-4}$ for mallard, $5.862 \times 10^{-4}$ for spotbilled duck, $5.815 \times 10^{-4}$ for Fenghua duck, $5.303 \times 10^{-4}$ for Shaoxing duck, $5.462 \times 10^{-4}$ for Shanma duck and $4.694 \times 10^{-4}$ for Cherry Valley Pekin duck (Supplementary Table S5), There values were muchlower than in other animals (Supplementary Table S6). The wild duck had the greatest $\theta_{\pi}$ and $\theta_{W}$, suggesting that domestication reduces genetic diversity. Additionally, Linkage disequilibrium (LD) also showed that the wild ducks had a faster decay of the pairwise correlation coefficient $\left(r^{2}\right)$ than the domestic duck (Fig. 1D).

\section{Demographic History}

We employed the pairwise sequentially Markovian coalescent (PSMC) method [12] to infer fluctuations in the ancestral effective population sizes $\left(N_{\mathrm{e}}\right)$ of each breed in response to Quaternary climatic change (Fig. 3A). From 1 million to 10 thousand years, all of the domestic breeds (SX, SM, CV and FH) exhibited similar demographic trajectories with a peak in ancestral $N_{\mathrm{e}}$ at 50-60 thousand years ago followed by distinct declines (Supplementary Fig. S3). The decline occurred $~ 60$ thousand year ago, coinciding with the beginning of the Last Glacil Maximum [37]. The effective population sizes of mallard and spot-billed duck appears to have increased rapidly after $\sim 40$ and $\sim 20$ thousand year ago, respectively (Supplementary Fig. S3).

\section{Genome-Wide Selective Sweep Test}

To accurately detect the genomic footprints of selection, we pooled the domestic duck samples (SX, SM and $\mathrm{CV}$ ) and compared them to the wild duck (MA and SB), which are geographically close. Using the top $5 \%$ the $F_{\mathrm{ST}}$ values and $\theta_{\pi}$ ratio cutoffs $\left(F_{\mathrm{ST}}>0.13\right.$ and $\log _{2}\left(\theta_{\pi}\right.$ ratio $\left.\left(\theta_{\pi, \text { wild duck }} / \theta_{\pi \text {, domestic duck }}\right) \geq 0.84\right)$, we identified 665 candidate domestication regions (CDRs) containing 387 genes under selection in the domestic ducks (Fig. 4, Fupplementary table S7). In addition, ten candidate genes (Cmip, Tmem132b, Mphosph6, Smg7, Lyst, Zbtb37, Serpinc1, Npl, Tmem132c and Plcg2) ranking within the top $10 F_{\mathrm{ST}}$ values with $\log _{2}\left(\theta_{\pi}\right.$ ratio $\left(\theta_{\pi \text {, wild duck }} / \theta_{\pi \text {, domestic duck }}\right) \geq 0.84$ were functionally involved in cellular adhesion function, type 2 diabetes, lipid metabolism, cell cycle, liver cell proliferation and muscle functioning [13-18] (Table 1). 
Table 1

Positively selected genes with top 10 FST values in domestic ducks.

\begin{tabular}{|c|c|c|c|c|}
\hline Gene ID & Gene name & $\begin{array}{l}\text { Gene } \\
\text { symbol }\end{array}$ & Fst & Description \\
\hline ENSAPLT00000011005 & $\begin{array}{l}\text { c-Maf inducing } \\
\text { protein }\end{array}$ & Cmip & 0.496 & $\begin{array}{l}\text { associating with language } \\
\text { and reading, type } 2 \text { diabetes, } \\
\text { obesity, lipid metabolism, } \\
\text { breast and gastric cancer, } \\
\text { negatively regulating T cell } \\
\text { signaling [40-45] }\end{array}$ \\
\hline ENSAPLT00000011847 & $\begin{array}{l}\text { transmembrane } \\
\text { protein } 132 B\end{array}$ & Tmem132b & 0.468 & $\begin{array}{l}\text { associating with excessive } \\
\text { daytime sleepiness [46] }\end{array}$ \\
\hline ENSAPLT00000002396 & $\begin{array}{l}\text { M-phase } \\
\text { phosphoprotein } 6\end{array}$ & Mphosph6 & 0.464 & $\begin{array}{l}\text { regulating cell cycle and } \\
\text { ovary development, } \\
\text { recruiting the exosome to } \\
\text { the pre-rRNA, associating } \\
\text { with coronary artery disease, } \\
\text { IgA nephropathy and } \\
\text { leukocyte telomere length } \\
\text { [47-51] }\end{array}$ \\
\hline ENSAPLT00000016529 & $\begin{array}{l}\text { nonsense } \\
\text { mediated mRNA } \\
\text { decay factor }\end{array}$ & Smg7 & 0.463 & $\begin{array}{l}\text { regulating DNA damage } \\
\text { response and nonsense- } \\
\text { mediated mRNA decay [52] }\end{array}$ \\
\hline ENSAPLT00000006672 & $\begin{array}{l}\text { lysosomal } \\
\text { trafficking } \\
\text { regulator }\end{array}$ & Lyst & 0.458 & $\begin{array}{l}\text { associating with Chediak- } \\
\text { Higashi syndrome [53] }\end{array}$ \\
\hline ENSAPLT00000005034 & $\begin{array}{l}\text { zinc finger and } \\
\text { BTB domain } \\
\text { containing } 37\end{array}$ & Zbtb37 & 0.424 & $\begin{array}{l}\text { involving in aryl } \\
\text { hydrocarbon receptor in } \\
\text { hematopoietic stem cell } \\
\text { functional regulation [54] }\end{array}$ \\
\hline ENSAPLT00000005105 & $\begin{array}{l}\text { serpin family C } \\
\text { member } 1\end{array}$ & Serpinc1 & 0.424 & $\begin{array}{l}\text { associating with } \\
\text { antithrombin deficiency and } \\
\text { ovarian cancer }[55,56]\end{array}$ \\
\hline ENSAPLT00000004292 & $\begin{array}{l}\mathrm{N}- \\
\text { acetylneuraminate } \\
\text { pyruvate lyase }\end{array}$ & $\mathrm{Npl}$ & 0.421 & $\begin{array}{l}\text { regulating the cellular } \\
\text { concentrations of sialic acid } \\
\text { which is essential for } \\
\text { muscle function [57] }\end{array}$ \\
\hline ENSAPLT00000012003 & $\begin{array}{l}\text { transmembrane } \\
\text { protein } 132 \mathrm{C}\end{array}$ & Tmem132c & 0.412 & $\begin{array}{l}\text { associating with pulmorary } \\
\text { function, breast cancer, } \\
\text { insulin secretion } \\
\text { impairment, body weight } \\
\text { [58-61] }\end{array}$ \\
\hline ENSAPLT00000011198 & $\begin{array}{l}\text { phospholipase C } \\
\text { gamma } 2\end{array}$ & Plcg2 & 0.391 & $\begin{array}{l}\text { involving in inherited } \\
\text { immune disorders, } \\
\text { promoting liver cell } \\
\text { proliferation [62-63] }\end{array}$ \\
\hline
\end{tabular}


To identify the active pathways in the domestication of ducks, the positively selected genes in domestic ducks were mapped to the canonical reference pathways in the KEGG database. The top three enriched pathways were "pantothenate and CoA biosynthesis" (2 genes, $P=0.02667)$, "FoxO signaling pathway" (6 genes, $P=0.03002$ ), and "inositol phosphate metabolism" (4 genes, $P=0.03511$ ) (Supplementary Fig. S4, Supplementary Table S8). The positively selected genes of domestic ducks that were successfully annotated to 47 categories of Gene Ontology (GO), belonging to three parts: cellular components, molecular function and biological processes (Supplementary Fig. S5, Supplementary Table S9). Of these, the categories that were most represented in the "biological process" principal category were "cellular process" (137 genes), followed by "single-organism process" (123 genes). In the principal category of "cellular component", the two categories most represented were "cell" (149 genes) and "cell part" (149 genes). Within the "molecular function" principal category belonged to the "bind" (107 genes).

\section{Positively Selected Genes Involved in Insulin Signaling Pathway}

Using the top $5 \%$ of the $F_{S T}$ values and $\theta \pi$ ratio cutoffs based on sliding $40 \mathrm{~kb}$ windows for the Shaoxing group compared to the mallard group, we identified 497 candidate domestication regions (CDRs) containing 311 genes with both high $F_{S T}$ values and a high $\theta \pi$ ratio (fig. 5A). Six genes exhibiting strong selective sweep signals were significantly over-represented in insulin signaling pathway, including ectonucleotide pyrophosphatase /phosphpdisesterase-1 (Enpp1), ectonucleotide pyrophosphatase/phosphpdisesterase-3 (Enpp3), SHC adapter protein 4 (Shc4), SOS Ras/Rac guanine nucleotide exchange factor 1 (Sos 1), neuroblastoma RAS viral oncogene homolog (Nras) and protein kinase cAMP-dependent type II regulatory subunit beta (Prkar2b).

Notably, we observed much higher $F_{\mathrm{ST}}$ values (fig. 5C) and lower Tajima's D values (fig. 5D) for the target gene Enpp 1 compared to those in the adjacent genomic regions, providing further support that the candidate genes were reliable. We also used transcriptome sequencing to investigate the molecular signatures of domestication and identified significantly downregulation Enpp1 expression in the muscle and liver tissues of Shaoxing ducks compared to mallards (fig. 5B).

\section{Discussion}

\section{Population structure and demographic history}

China has the largest number of ducks in the world and was one of the earliest to domesticate wild ducks $[19,20]$. However, the origin of Chinese domestic ducks is still controversial. Here, we advance a new hypothesis that the domesticated ducks were not direct descendants of the mallard or Spot-billed duck and probably originated from an unknown population on the basis of the whole-genome sequences. 
In this study, we carried out whole-genome resequencing of 60 individuals to explore the genetic relationships among the domestic ducks and wild ducks in eastern China. The PCA and NJ tree results suggest that mallard and spot-billed duck cluster more closely with each other and have a closer genetic relationship. This finding raised a question as to whether Chinese domestic ducks originate from mallard and spot-billed duck. In order to solve this problem, we perform population genetic structure analysis.. A surprising result was the clear division between wild ducks and domestic breeds in the clustering analysis when $K=2$, and spot-billed duck and mallard were separated only from $K=5$. This suggests that the split of mallard and spot-billed duck are later than that of Chiese domestic ducks. Using TreeMix to infer migration patterns among the different populations, we did not find any gene flow among wild ducks, Shaoxing duck, Shanma duck and Cherry Valley Pekin duck, but this analysis show the strong gene flow from Fenghua duck into mallard. It is speculated that the locations where Fenghua duck and mallard were collected contribute to this gene flow.

In the PSMC model, we observed the similar trajectories for four Chinese domestic ducks with an aparent expansion during the Penultimate Glaciation and the Last Interglaciation, and a decline between 50 and 60 thousand years ago. In addition, mallard and spot-billed duck population reached their pinnacle between 20 and 40 thousand years ago. This is consistent with our population genetic structure results that the ancestor of Chinese domestic ducks is different from the ancestors of mallard and spot-billed duck.

\section{Selection for domestication}

The Enpp1, Enpp3, Shc4, Sos1, Nras and Prkar2b are related to the insulin signaling pathway and showed signals of positive selection in Shaoxing duck. Enpp 1 is the subtypes of ENPP family, which directly interacts with the insulin receptor and blocks the insulin signaling pathway [21], serving as a gatekeeper of insulin action. ENPP3 is positive associated with the serum ATP concentration, facilitating lipid deposition [22]. The protein encoded by Prkar $2 b$ is a regulatory subunits of the protein kinase A ( $P K A)$ and is involved in insulin resistance [23]. Shc4 (also known as ShcD) serves as a phosphotyrosine adapter molecule that induces Ras GTPase and mitogen activated protein kinase (MAPK) activation [24]. Also, the SOS1 and NRAS provide protein-making instructions that are involved in regulating the activation of the Ras/MAPK signaling pathway, which helps to control insulin signaling (fig. 6).

These genes may have played a crucial role in duck domestication by altMangmoolering glucose and lipid metabolism. Differences in glucose and lipid metabolism between wild and domesticated animals due to directional selection were also observed in pigs [25] and chickens [3]. Enpp3 and Prkar2b were identified as the targets of selection during the domestication of Pekin ducks and other indigenous Chinese ducks [26,27].

\section{Conclusion}


In conclusion, we performed whole-genome resequencing to characterize the evolutionary origin of ducks in eastern China and the genome-wide signatures of artificial selection associated with domestication.

Several candidate genes, important pathways and GO categories associated with artificial selection were functionally related to cellular adhesion, type 2 diabetes, lipid metabolism, the cell cycle, liver cell proliferation, and muscle functioning in domestic ducks. We found strong genomic evidence for the involvement of the insulin signaling pathway in the domestication of Shaoxing duck. These results advance our understanding of the genetic relationships between domestic and wild ducks, reveals the genetic footprints of domestication and shed light on the genetic mechanisms underlying species adaptation to captivity.

\section{Methods}

\section{Sampling}

The blood samples from all the 60 individual ducks ( 10 per breed) were collected from the wing vein using vacuum tubes containing EDTA- $\mathrm{K}_{2}$ as an anticoagulant. The spot-billed ducks, mallards and Fenghua ducks were captured in Fenghua City, Zhejiang Province, China $\left(29^{\circ} 35^{\prime} \mathrm{N}, 121^{\circ} 24^{\prime} \mathrm{E}\right)$. The Shaoxing and Shanma ducks were collected in Zhuji City, Zhejiang Province, China ( $\left.29^{\circ} 38^{\prime} \mathrm{N}, 120^{\circ} 10^{\prime} \mathrm{E}\right)$, and the Cherry Valley Pekin duck were raised in Huzhou City, Zhejiang Province, China $\left(30^{\circ} 41^{\prime} \mathrm{N}, 120^{\circ} 19^{\prime}\right.$ E). From Shaoxing ducks and mallards, 3 randomly selected ducklings were killed by rapid decapitation and sterile dissection, and muscle and liver tissues were sampled and immediately snap frozen in liquid nitrogen.

\section{Sequencing and Quality Control}

A total of 60 ducks, which were sampled from Eastern China, were sequenced on the Illumina HiSeq 2000 platform (Illumina, San Diego CA, USA). We generated a total of $401.491 \mathrm{~Gb}$ of raw sequence data (supplementary table S1).

To make sure reads reliable and without artificial bias in the following analyses, raw reads of fastq format were firstly processed through a series of quality control (QC) procedures in-house $C$ scripts. The low-quality reads were filtered out based on the following [28]:

(1) Reads with $\geq 10 \%$ unidentified nucleotides $(N)$;

(2) Reads with $>50 \%$ bases having phred quality $<5$;

(3) Reads with >10 nt aligned to the adapter, allowing $\leq 10 \%$ mismatches;

(4) Putative PCR duplicates generated by PCR amplification in the library construction process (read 1 and read 2 of two paired-end reads that were completely identical). 
Consequently, $387.88 \mathrm{~Gb}$ were retained for assembly, of which the quality of $96.06 \%$ and $91.52 \%$ of the bases were $\geq Q 20$ and $\geq Q 30$, respectively.

\section{Reads Mapping and SNP Calling}

The remaining high quality reads were mapped to the mallard (Anas platyrhynchos) reference genome [29] using Burrows-Wheeler Aligner (Version: 0.7.8) [30] with the command line was 'BWA mem -t 4 -k 32 $-M$ '. SAMtools was used to remove the duplicated reads to reduce mismatch generated by PCR amplification before sequencing.

After alignment, we used SAMtools [31] to carry out SNP calling. The 'mpileup' command was used to identify SNPs with the parameters as '-q 1 -C 50 -S -D -m 2-F 0.002'. The following filtering steps were applied in order to obtain high quality SNPs:

(1) Quality score > = 20 .

(2) Coverage depth $>=2$ and $<=1000$.

\section{Annotation of Genetic Variants}

Using the ANNOVAR package [32], 2,809,077 high-quality SNPs were annotated according to the genome. Based on the genome annotation, SNPs were classified into several categories, such as exonic regions, intronic regions, splicing sites, upstream and downstream regions and intergenic regions. The SNPs in coding exons were fall into two categories: synonymous SNPs and nonsynonymous SNPs.

\section{Principal Component Analysis}

The software GCTA [33] was used for PCA with the population scale SNPs. The significance level of the eigenvectors was determined using the Tracey-Widom test to clarify the phylogenetic relationship among 60 individuals. The first three significant components were plotted (supplementary fig. S2), and the discrete points to a degree reflect the real structure of population.

\section{Phylogenetic Tree}

We also inferred an individual-based neighbor-joining $(\mathrm{NJ})$ tree in TreeBeST (http://treesoft.sourceforge.net/treebest.shtml\#inno) based on the $p$-distance. The bootstrap was set to 1,000 times to evaluate the reliability of branch..

\section{Population Structure and TreeMix analysis}


The population genetic structure of 60 individuals was inferred by FRAPPE [34]. We set the number of cluster (K) from 2 to 6 and ran analysis with 10,000 iterations. Migration events among the populations were inferred using TreeMix [35]. We converted plink output for TreeMix by plink2treemmix module in TreeMix software. We set the number of migration events from 1 to 3 . For each number of migration events, we ran 100 random input orders.

\section{Linkage Disequilibrium Analysis}

We compared the pattern of linkage disequilibrium (LD) among 6 breeds using the 2.8 million high-quality SNPs. To estimate LD decay, we calculated the squared correlation coefficient $\left(r^{2}\right)$ between pairwise SNPs using the software Haploview [36]. The average $r^{2}$ value was calculated for pairwise markers in a 500-kb window and averaged across the whole genome.

\section{Effective Population Size}

We used a hidden Markov model (HMM) of pairwise sequentially Markovian coalescence (PSMC) to reconstructed demographic history of 60 individuals. Firstly, we called genotype each individual using the package SamTools [30] based on the command 'mpileup' with the parameter '-C 50 -D -S -m 2 -F 0.002'. Then, we performed the program ' $f q 2 p s m c f a$ ' with the parameter ' $-N 30,-t 15,-r 5$ and $-p$ ' $4+25 * 2+4+6$ " to convert the consensus sequence to the required input format. A mutation rate $(\mu)$ of $1.6 \times 10^{-9}$ per bp per generation [7] and a generation time of 1 year were used for analysis. In addition, we applied a bootstrapping approach, repeating sampling 100 times on two samples to estimate the variance of simulated results.

\section{Selective Sweep Analysis}

The nucleotide diversity $\left(\theta_{\pi}\right)$, population-differentiation statistic $\left(F_{\mathrm{ST}}\right)$, Tajima's $D$ statistic and Watterson estimator $\left(\theta_{W}\right)$ were calculated with sliding windows of $40 \mathrm{~kb}$ that had $20 \mathrm{~kb}$ overlap between adjacent windows. The putative genomic regions under positive selection during domestication were extracted based on being the highest differences in genetic diversity $\left(\log _{2}\left(\theta_{\pi}\right.\right.$ ratio $\left.)\right)$ and the top $5 \%$ of $F_{\mathrm{ST}}$. We identified a total of 665 potential selective-sweep regions overlapping with 387 candidate genes in merging domestic ducks and 491 potential selective-sweep regions overlapping with 311 candidate genes in Shaoxing ducks, which would be used for subsequent analysis and discussion.

\section{Functional Enrichment Analysis}

Gene Ontology term enrichment analysis was processed with those selective genes by goseq packages in $\mathrm{R}$ software. We used the GOSeq R package, in which gene length bias was corrected, to perform GO and 
functional pathway analysis on the candidate genes. The Gene ontology (GO) and the Kyoto Encyclopedia of Genes and Genomes (KEGG) pathways with a Benjamini adjusted $P$-values less than 0.05 were considered significantly enriched.

\section{RNA-seq and Gene Expression Analysis}

To infer whether the genes under selection could also affecting gene expression between Shaoxing ducks and mallards, we compared gene expression in breast muscle, liver and cerebellum between this two groups. 3 females from Shaoxing ducks and mallards respectively were selected for transcriptomics analysis.

All samples were individually sequenced by Illumina HiSeq 4000 sequencing platform. Perl scripts was used to ensure the quality of raw data. The reference genomes and the annotation file were downloaded from ENSEMBL database (http://www.ensembl.org/index.html). We used Bowtie/Bowtie 2 to build the genome index and TopHat v2.0.12 to map clean data to reference genome. And HTSeq v6.0 was used to count the number of fragments for each gene in each sample. The expression level of genes in each sample was estimated by FPKM (Fragments Per Kilobase Per Million Mapped Fragment). We used DEGseq v1.18.0 to analyze differential gene expression between Shaoxng and Shanma ducks. Genes with $\mathrm{q} \leq 0.05$ and $\| \log _{2}$ Ratio| $\geq 1$ are identified as differentially expressed genes.

\section{Abbreviations}

YBP: years before present; SB: spot-billed duck, MA: mallard, FH: Fenghua duck, SX: Shaoxing duck, SM: Shanma duck, CV: Cherry Valley Pekin duck, SNP: single nucleotide polymorphic site, PCA: principal component analysis, NJ tree: neighbor-joining tree, PSMC: pairwise sequentially Markovian coalescent, GO: gene ontology, CDR: candidate domestication region, Enpp1: ectonucleotide pyrophosphatase /phosphpdisesterase-1, Enpp3: ectonucleotide pyrophosphatase/phosphpdisesterase-3, Shc4: SHC adapter protein 4, Sos1: Ras/Rac guanine nucleotide exchange factor 1, Nras: neuroblastoma RAS viral oncogene homolog, Prkar2b: protein kinase cAMP-dependent type II regulatory subunit beta.

\section{Declarations}

\section{Ethics approval and consent to participate}

The animal care and protocol used in this study were reviewed and approved by the Insitutional Animal Care and Use Committee of the Zhejiang Academy of Agricultural Sciences and South China Agricultural University, and performed in accordance with the Regulations for the Administration of Affairs Concerning Experimental Animals (SCAU-10564).

\section{Consent for publication}




\section{Avalability of data and material}

The raw sequence data files discussed in this experiment have been deposited in SRA and BioProject ID is PRJNA599025 (https://dataview.ncbi.nlm.nih.gov/object/PRJNA599 025)

\section{Competing interests}

We certify that there is no conflict of interest with any financial organization regarding the material discussed in the manuscript.

\section{Founding}

This work was sponsored by the earmarked fund for National Waterfowl-industry Technology Research System (CARS-42-6 and CARS-42-15) and the Natural Science Foundation of China (31702106). The funders had no role in study design, data collection and analysis, decision to publish, or preparation of the manuscript.

\section{Authors' contributions}

PF analyzed main content of the data and performed the experiment with the help of HY, PW and LC. TZ collected samples with the help of JD, JS, ZT and GC The whole work is guided by LY and LL. All authors read and approved the final manuscript.

\section{Acknowledgements}

Thank Ningbo Aoji Agricultural Science and Technology Co. LTD, Zhejiang Zhuoan Poultry Co. LTD, and Guowei Poultry Industry Co, LTD for their great help in sampling.

\section{References}

1. Price EO. Behavioral aspects of animal domestication. Q Rev Biol. 1984;59(59):1-32. doi:10.1086/413673.

2. Li M, Tian S, Yeung CK, Meng X, Tang Q, Niu L, et al. Whole-genome sequencing of Berkshire (European native pig) provides insights into its origin and domestication. Sci Rep. 2014;4(4):4678. doi:10.1038/srep04678. 
3. Rubin CJ, Zody MC, Eriksson J, Meadows JR, Sherwood E, Webster MT, et al. Whole-genome resequencing reveals loci under selection during chicken domestication. Nature. 2010;464(7288):587-91. doi:10.1038/nature08832.

4. Axelsson E, Ratnakumar A, Arendt ML, Maqbool K, Webster MT, Perloski M, et al. The genomic signature of dog domestication reveals adaptation to a starch-rich diet. Nature. 2013;495(7441):360. doi:10.1038/nature11837.

5. Qiang Q, Wang L, Wang K, Yang Y, Tao M, Wang Z, et al. Yak whole-genome resequencing reveals domestication signatures and prehistoric population expansions. Nat Commun. 2015;6:10283. doi:10.1038/ncomms10283.

6. Larson G, Fuller DQ. The evolution of animal domestication. Annu Rev Ecol Evol Syst. 2014;45(3):115-36. doi:10.1146/annurev-ecolsys-110512-135813.

7. Zhang Y, Chen Y, Zhen T, Huang Z, Chen C, Li X, et al. Analysis of the Genetic Diversity and Origin of Some Chinese Domestic Duck Breeds. J Integr Agr. 2014;13(4):849-57. doi:10.1016/S20953119(13)60447-5.

8. Li H, Zhu W, Song W, Shu J, Han W, Chen K. Origin and genetic diversity of Chinese domestic ducks. Mol Phylogenet Evol. 2010;57(2):634-40. doi:10.1016/j.ympev.2010.07.011.

9. Guay P, Iwaniuk AN. Captive breeding reduces brain volume in waterfowl (Anseriformes). Condor. 2008;110(2):276-84. doi:10.1525/cond.2008.8424.

10. Duggan BM, Hocking PM, Schwarz T, Clements DN. Differences in hindlimb morphology of ducks and chickens: effects of domestication and selection. Genet Sel Evol. 2015;47:88. doi:10.1186/s12711-015-0166-9.

11. Yang S, Zhou L, Lin W, Li X, Lu M, Liu C. Behavioral differentiation between Anas poecilorhyncha and domestic duck. J Agr Sci Tech-iran. 2016;4:270-82. doi:10.17265/2161-6256/2016.04.007.

12. Li H, Durbin R. Inference of human population history from individual whole-genome sequences. Nature. 2011;475(7357):493-6. doi:10.1038/nature10231.

13. Ling H, Waterworth DM, Stirnadel HA, Pollin TI, Barter PJ, Kesäniemi YA, et al. Genome-wide linkage and association analyses to identify genes influencing adiponectin levels: the gems stud. Obesity. 2009;17(4):737-44. doi:10.1038/oby.2008.625.

14. Zhou J, Qiu L, Jiang S, Zhou F, Huang J, Yang L, et al. Molecular cloning and mRNA expression of Mphase phosphoprotein 6 gene in black tiger shrimp (Penaeus monodon). Mol Biol Rep. 2013;40(2):1301-6. doi:10.1007/s11033-012-2173-z.

15. Keaton JM, Gao C, Guan M, Hellwege JN, Palmer ND, Pankow JS, et al. Genome-wide interaction with the insulin secretion locus MTNR1B reveals CMIP as a novel type 2 diabetes susceptibility gene in African Americans. Genet Epidemiol. 2018;42(6):559-70. doi:10.1002/gepi.22126.

16. Sanchez-Pulido L, Ponting CP. TMEM132: an ancient architecture of cohesin and immunoglobulin domains define a new family of neural adhesion molecules. Bioinformatics. 2018;34(5):721-4. doi:10.1093/bioinformatics/btx689. 
17. Wen X, Tarailo-Graovac M, Brand-Arzamendi K, Willems A, Rakic B, Huijben K, et al. Sialic acid catabolism by $\mathrm{N}$-acetylneuraminate pyruvate lyase is essential for muscle function. JCl Insight. 2018;3(24):122373. doi:10.1172/jci.insight.122373.

18. Ma D, Lian F, Wang X. PLCG2 promotes hepatocyte proliferation in vitro via NF-KB and ERK pathway by targeting bcl2, myc and ccnd1. Artif Cells Nanomed Biotechnol. 2019;47(1):3786-92. doi:10.1080/21691401.2019.1669616.

19. CASS (Chinese Academy of Social Sciences). (1979). Archaeological perspectives on Yin ruins, Anyang excavations in 1969-1977, China. Acta Archaeological Sinica, Institute of Archaeology 1.

20. Kenneth FK, Kriemhild CO. The Cambridge world history of food. Cambridge: Cambridge University Press, 2000.

21. Maddux BA, Goldfine ID. Membrane glycoprotein PC-1 inhibition of insulin receptor function occurs via direct interaction with the receptor alpha-subunit. Diabetes. 2000;49(1):13-9. doi:10.2337/diabetes.49.1.13.

22. Tsai SH, Kinoshita M, Kusu T, Kaama H, Okumura R, lkeda K, et al. The ectoenzyme E-NPP3 negatively regulates ATP-dependent chronic allergic responses by basophils and mast cell. Immunity. 2015;42(2):279-93. doi:10.1016/j.immuni.2015.01.015.

23. $10.1210 / m e .2015-1201$

Mangmool S, Denkaew T, Phosri S, Pinthong D, Parichatikanond W, Shimauchi T, et al., (2016). Sustained $\beta A R$ Stimulation Mediates Cardiac Insulin Resistance in a PKA-Dependent Manner. Mol. Endocrino./30(1): 118-132. doi: 10.1210/me.2015-1201.

24. Pasini L, Lanfrancone L. SHC4 (SHC (Src homology 2 domain containing) family, member 4). Atlas Genet Cytogenet Oncol Haematol. 2011;14(8):732-4. doi:10.4267/2042/44817.

25. Li X, Yang S, Dong K, Tang A, Li K, Fan B, et al. Identificaton of positive selection signatures in pigs by comparing linkage disequilibrium variances. Anim Genet. 2017;48(5):600-5. doi:10.1111/age.12574.

26. Wang L, Li X, Ma J, Zhang Y, Zhang H. Integrating genome and transcriptome profiling for elucidating the mechanism of muscle growth and lipid deposition in Pekin ducks. Sci Rep. 2017;7(1):3837. doi:10.1038/s41598-017-04178-7.

27. Zhang Z, Jia Y, Almeida P, Mank JE, Tuinen M, Wang Q, et al., (2018).Whole-genome resequencing reveals signatures of selection and timing of duck domestication. GigaScience 7(4). doi:10.1093/gigascience/giy027.

28. Chen C, Liu Z, Pan Q, Chen X, Wang H, Guo H, et al. Genomic analyses reveal demographic history and temperate adaptation of the newly discovered honey bee subspecies Apis mellifera sinisxinyuan n. ssp. Mol Biol Evol. 2016;33(5):1337-48. 10.1093/molbev/msw017. doi.

29. Huang Y, Li Y, Burt DW, Chen H, Zhang Y, Qian W, et al. The duck genome and transcriptome provide insight into an avian influenza virus reservoir species. Nat Genet. 2013;45(7):776-83. doi:10.1038/ng.2657. 
30. Li H, Durbin R. Fast and accurate short read alignment with Burrows-Wheeler transform. Bioinformatics. 2009;25(14):1754-60. doi:10.1093/bioinformatics/btp324.

31. Li H, Handsaker B, Wysoker A, Fennell T, Ruan J, Homer N, et al. The Sequence Alignment/Map format and SAMtools. Bioinformatics. 2009;25(16):2078-9. doi:10.1093/bioinformatics/btp352.

32. Wang K, Li M, Hakonarson H. ANNOVAR: functional annotation of genetic variants from highthroughput sequencing data. Nucleic Acids Res. 2010;38(16):e164. doi:10.1093/nar/gkq603.

33. Yang J, Lee SH, Goddard ME, Visscher PM. GCTA: A tool for genome-wide complex trait analysis. Am J Hum Genet. 2011;88(1):76-82. doi:10.1016/j.ajhg.2010.11.011.

34. Tang H, Quertermous T, Rodriguez B, Kardia SLR, Zhu X, Brown A, et al. Genetic structure, selfIdentified race/ethnicity, and confounding in case-control association studies. Am J Hum Genet. 2005;76(2):268-75. doi:10.1086/427888.

35. Pickrell JK, Pritchard JK. Inference of population splits and mixtures from genome-wide allele frequency data. PLoS Genet. 2012;8:e1002967. doi:10.1371/journal.pgen.1002967.

36. Barrett JC, Fry B, Maller J, Daly MJ. Haploview: analysis and visualization of LD and haplotype maps. Bioinformatics. 2005;21(2):263-5. doi:10.1093/bioinformatics/bth457.

37. Zheng B, Xu Q, Shen Y. (2002). The relationship between climate change and Quaternary glacial cycles on the Qinghai-Tibetan Plateau: review and speculation. Quatern Int 97 - 8(1): 93-101. doi:10.1016/S1040-6182(02)00054-X.

38. Bintanja R, van de Wal RSW, Oerlemans J. Modelled atmospheric temperatures and global sea levels over the past million years. Nature. 2005;437(7055):125-8. doi:10.1038/nature03975.

39. Zhou Z, Li M, Cheng H, Fan W, Yuan Z, Gao Q, et al., (2018). An intercross population study reveals genes associated with body size and plumage color in ducks. Nat Commun 9: 2648. doi: 10.1038/s41467-018-04868-4 |www.nature.com/naturecommunications.

40. Keaton JM, Gao C, Guan M, Hellwege JN, Palmer ND, Pankow JS, et al. Genome-wide interaction with the insulin secretion locus MTNR1B reveals CMIP as a novel type 2 diabetes susceptibility gene in African Americans. Genet Epidemiol. 2018;42(6):559-70. doi:10.1002/gepi.22126.

41. Ling H, Waterworth DM, Stirnadel HA, Pollin TI, Barter PJ, Kesäniemi YA, et al. Genome-wide linkage and association analyses to identify genes influencing adiponectin levels: the gems stud. Obesity. 2009;17(4):737-44. doi:10.1038/oby.2008.625.

42. Newbury DF, Gibson JL, Conti-Ramsden G, Pickles A, Durkin K, Toseeb U. Using polygenic profiles to predict variation in language and psychosocial outcomes in early and middle childhood. J Speech Lang Hear Res. 2019;62(9):3381-96. doi:10.1044/2019_JSLHR-L-19-0001.

43. Oniszczuk J, Sendeyo K, Chhuon C, Savas B, Cogne E, Vachin P, et al. CMIP is a negative regulator of T cell signaling. Cell Mol Immunol. 2019. doi:10.1038/s41423-019-0266-5.

44. Wang C, Li CY, Cai JH, Sheu PC, Tsai J, Wu MY, et al. Identification of prognostic candidate Genes in breast cancer by integrated bioinformatic analysis. J Clin Med. 2019;8(8):1160. doi:10.3390/jcm8081160. 
45. Zhang J, Huang J, Wang X, Chen W, Tang Q, Fang M, et al. CMIP is oncogenic in human gastric cancer cells. Mol Med Rep. 2017;16(5):7277-86. doi:10.3892/mmr.2017.7541.

46. Lane JM, Liang J, Vlasac I, Anderson SG, Bechtold DA, Bowden J, et al. Genome-wide association analyses of sleep disturbance traits identify new loci and highlight shared genetics with neuropsychiatric and metabolic traits. Nat Genet. 2017;49(2):274-81. doi:10.1038/ng.3749.

47. Zhou J, Qiu L, Jiang S, Zhou F, Huang J, Yang L, et al. Molecular cloning and mRNA expression of Mphase phosphoprotein 6 gene in black tiger shrimp (Penaeus monodon). Mol Biol Rep. 2013;40(2):1301-6. doi:10.1007/s11033-012-2173-z.

48. Dorajoo R, Chang X, Gurung RL, Li Z, Wang L, Wang R, et al., (2019). Loci for human leukocyte telomere length in the Singaporean Chinese population and trans-ethnic genetic studies. Nat Commun 10(1). doi:10.1038/s41467-019-10443-2.

49. Schilders G. MPP6 is an exosome-associated RNA-binding protein involved in 5.8S rRNA maturation. Nucleic Acids Res. 2005;33(21):6795-804. doi:10.1093/nar/gki982.

50. Song Y, Yan M, Li J, Li J, Jin T, Chen C. Association between TNIP1, MPHOSPH6 and ZNF208 genetic polymorphisms and the coronary artery disease risk in Chinese Han population. Oncotarget. 2017;8(44):77233. doi:10.18632/oncotarget.20432.

51. Yang X, Zhang Y, Li W, Su Y, Niu D, Wang Y, et al. Association between MPHOSPH6 gene polymorphisms and IgA nephropathy risk in a Chinese Han population. Oncotarget. 2017;8(42):72375. doi:10.18632/oncotarget.19758.

52. Luo H, Cowen $L$, Yu G, Jiang W, Tang Y. SMG7 is a critical regulator of $p 53$ stability and function in DNA damage stress response. Cell Discov. 2016;2(1):15042. doi:10.1038/celldisc.2015.42.

53. Sepulveda FE, Burgess A, Heiligenstein X, Goudin N, Ménager MM, Romao M, et al. LYST controls the biogenesis of the endosomal compartment required for secretory lysosome function. Traffic. 2015;16(2):191-203. doi:10.1111/tra.12244.

54. Bennett JA, Singh KP, Unnisa Z, Welle SL, Gasiewicz TA. Deficiency in aryl hydrocarbon receptor (AHR) expression throughout aging alters gene expression profiles in murine long-term hematopoietic stem cells. Plos One. 2015;10(7):e133791. doi:10.1371/journal.pone.0133791.

55. Kovac M, Mitic G, Djilas I, Kuzmanovic M, Serbic O, Lekovic D, et al. Genotype phenotype correlation in a pediatric population with antithrombin deficiency. Eur J Pediatr. 2019;178(10):1471-8. doi:10.1007/s00431-019-03433-5.

56. Zhang W, Peng P, Ou X, Shen K, Wu X. Ovarian cancer circulating extracelluar vesicles promote coagulation and have a potential in diagnosis: an ITRAQ based proteomic analysis. Bmc Cancer. 2019;19(1):1095. doi:10.1186/s12885-019-6176-1.

57. Wen X, Tarailo-Graovac M, Brand-Arzamendi K, Willems A, Rakic B, Huijben K, et al. Sialic acid catabolism by $\mathrm{N}$-acetylneuraminate pyruvate lyase is essential for muscle function. JCl Insight. 2018;3(24):122373. doi:10.1172/jci.insight.122373.

58. De Almeida BP, Apolónio JD, Binnie A, Castelo-Branco P. (2019). Roadmap of DNA methylation in breast cancer identifies novel prognostic biomarkers. Bmc Cancer 19(1). doi:10.1186/s12885-019- 
5403-0.

59. Lane JM, Liang J, Vlasac I, Anderson SG, Bechtold DA, Bowden J, et al. Genome-wide association analyses of sleep disturbance traits identify new loci and highlight shared genetics with neuropsychiatric and metabolic traits. Nat Genet. 2017;49(2):274-81. doi:10.1038/ng.3749.

60. Son H, Sohn S, Im S, Kim H, Lee MK, Gombojav B, et al. Family-Based association study of pulmonary function in a population in Northeast Asia. Plos One. 2015;10(10):e139716. doi:10.1371/journal.pone.0139716.

61. Gong H, Xiao S, Li W, Huang T, Huang X, Yan G, et al. Unravelling the genetic loci for growth and carcass traits in Chinese Bamaxiang pigs based on a 1.4 million SNP array. J Anim Breed Genet. 2019;136(1):3-14. doi:10.1111/jbg.12365.

62. Ma D, Lian F, Wang X. PLCG2 promotes hepatocyte proliferation in vitro via NF-KB and ERK pathway by targeting bcl2, myc and ccnd1. Artif. Cells Nanomed Biotechnol. 2019;47(1):3786-92. doi:10.1080/21691401.2019.1669616.

63. Ombrello MJ, Remmers EF, Sun G, Freeman AF, Datta S, Torabi-Parizi P, et al. Cold urticaria, immunodeficiency, and autoimmunity related to PLCG2 deletions. N Engl J Med. 2012;366(4):330-8. doi:10.1056/NEJMoa1102140.

64. Siddle K. Signalling by insulin and IGF receptors: supporting acts and new players. J Mol Endocerinol. 2011;47(1):1-10. doi:10.1530/JME-11-0022.

65. Mangmool S, Denkaew T, Parichatikanond W, et al. $\beta$-Adrenergic Receptor and Insulin Resistance in the Heart. Biomol Ther. 2017;25(1):44-56. doi:10.4062/biomolther.2016.128.

\section{Figures}




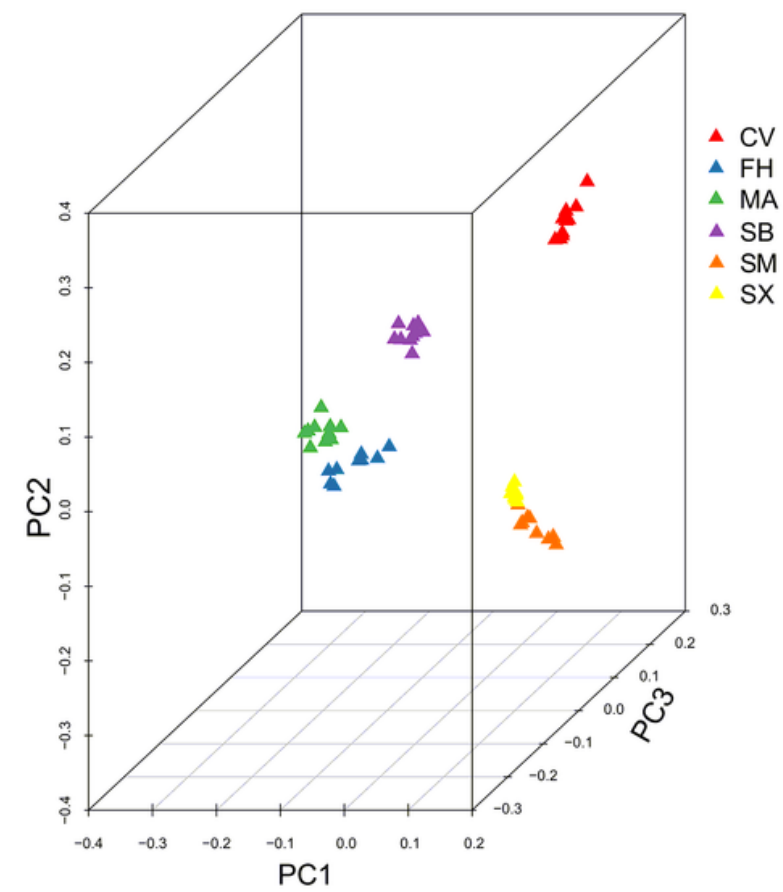

C

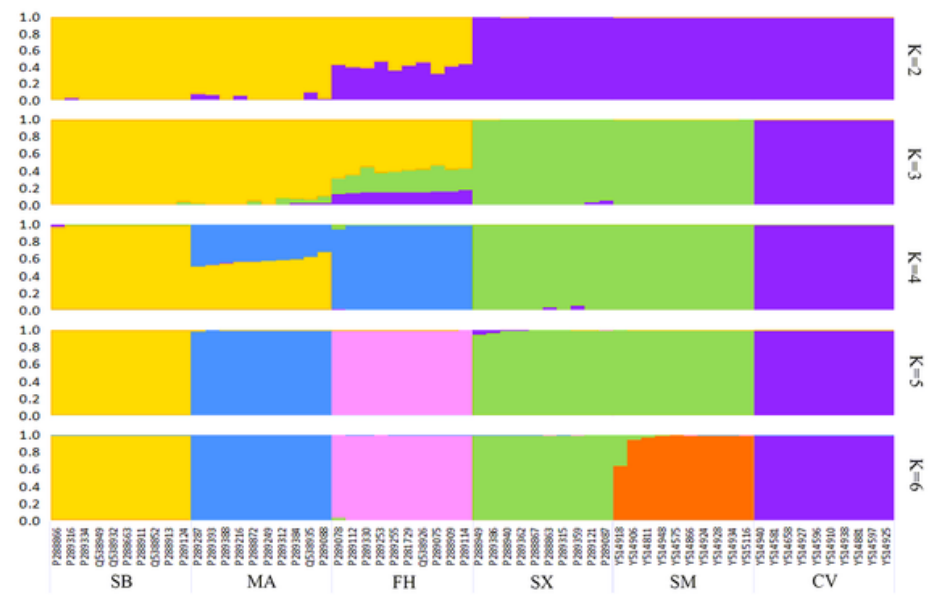

B

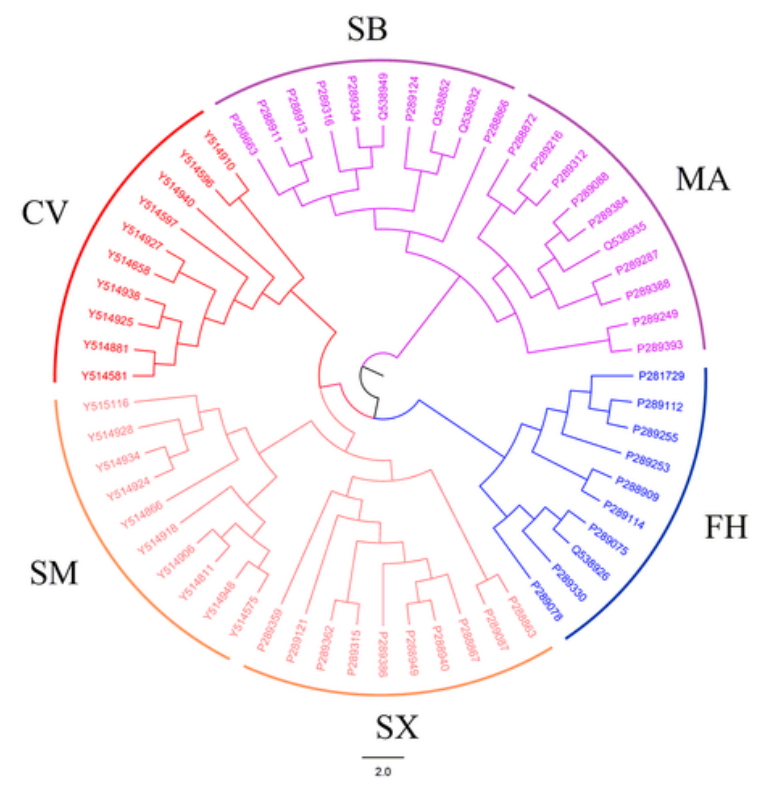

D

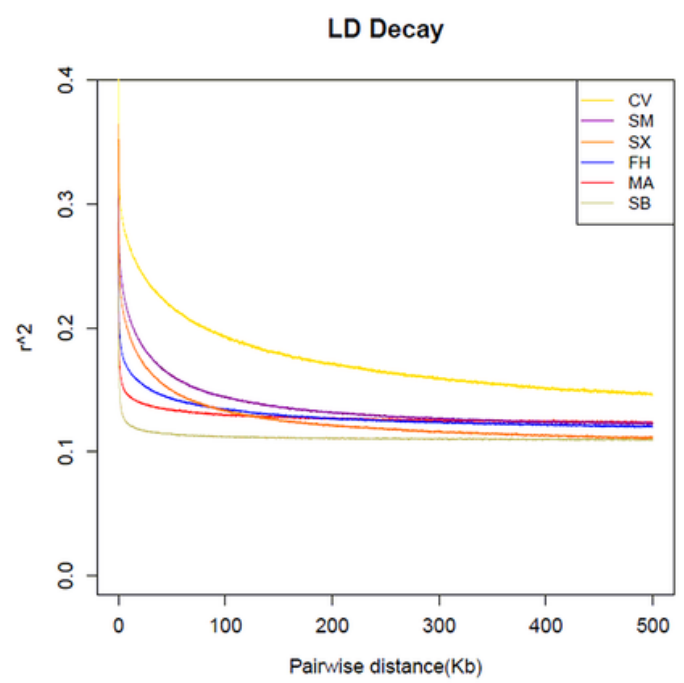

\section{Figure 1}

Phylogenetic and population genetic analyses of wild and domestic ducks. (A) Principal component plot of 60 individuals. (B) Unrooted neighbor-joining tree constructed using the p-distances between individuals. (C) Population structure of 60 ducks $(K=2-6)$. The $y$-axis represents the proportion of the individual's genome from inferred ancestral populations, and $x$-axis represents the different populations. (D) Genome-wide linkage disequilibrium of ducks. 

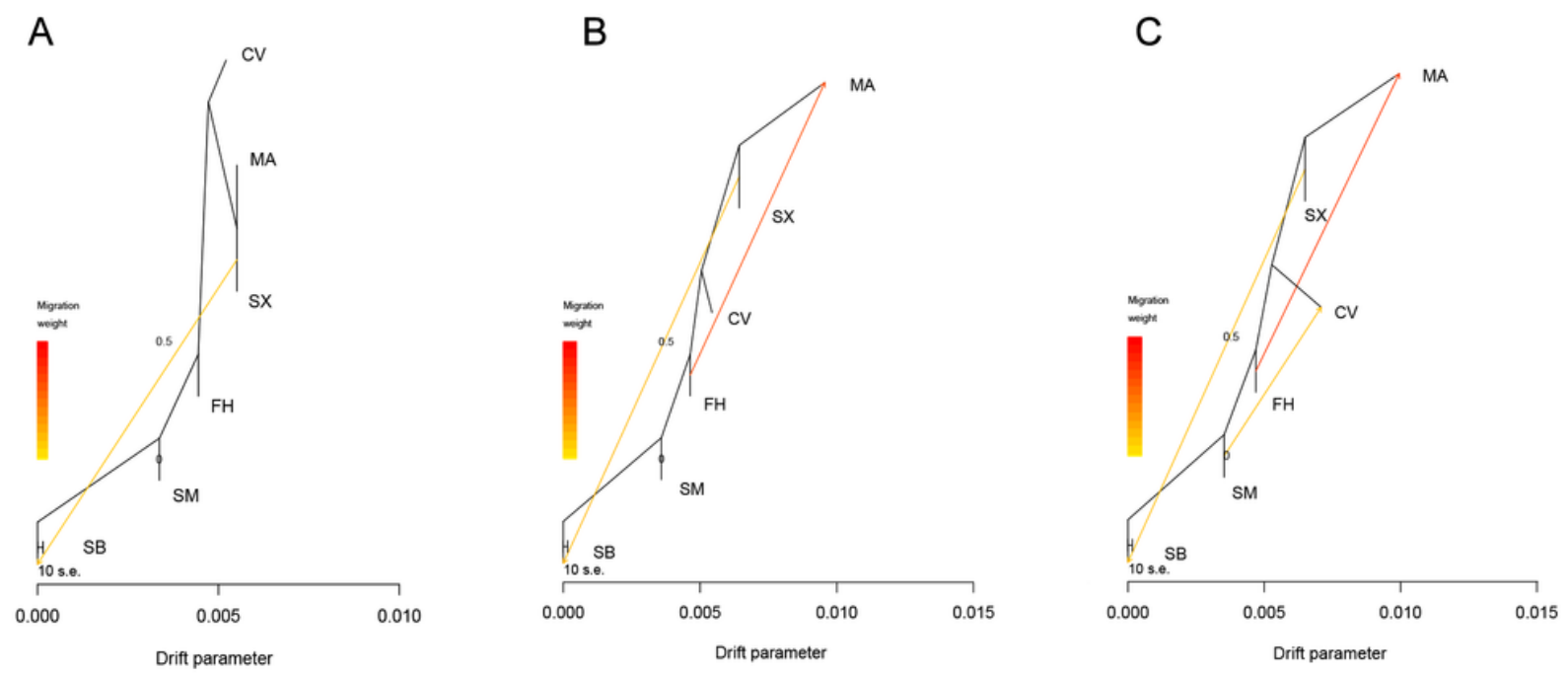

Figure 2

TreeMix analysis with (A) one, $(B)$ two and $(C)$ thtree migration events. 


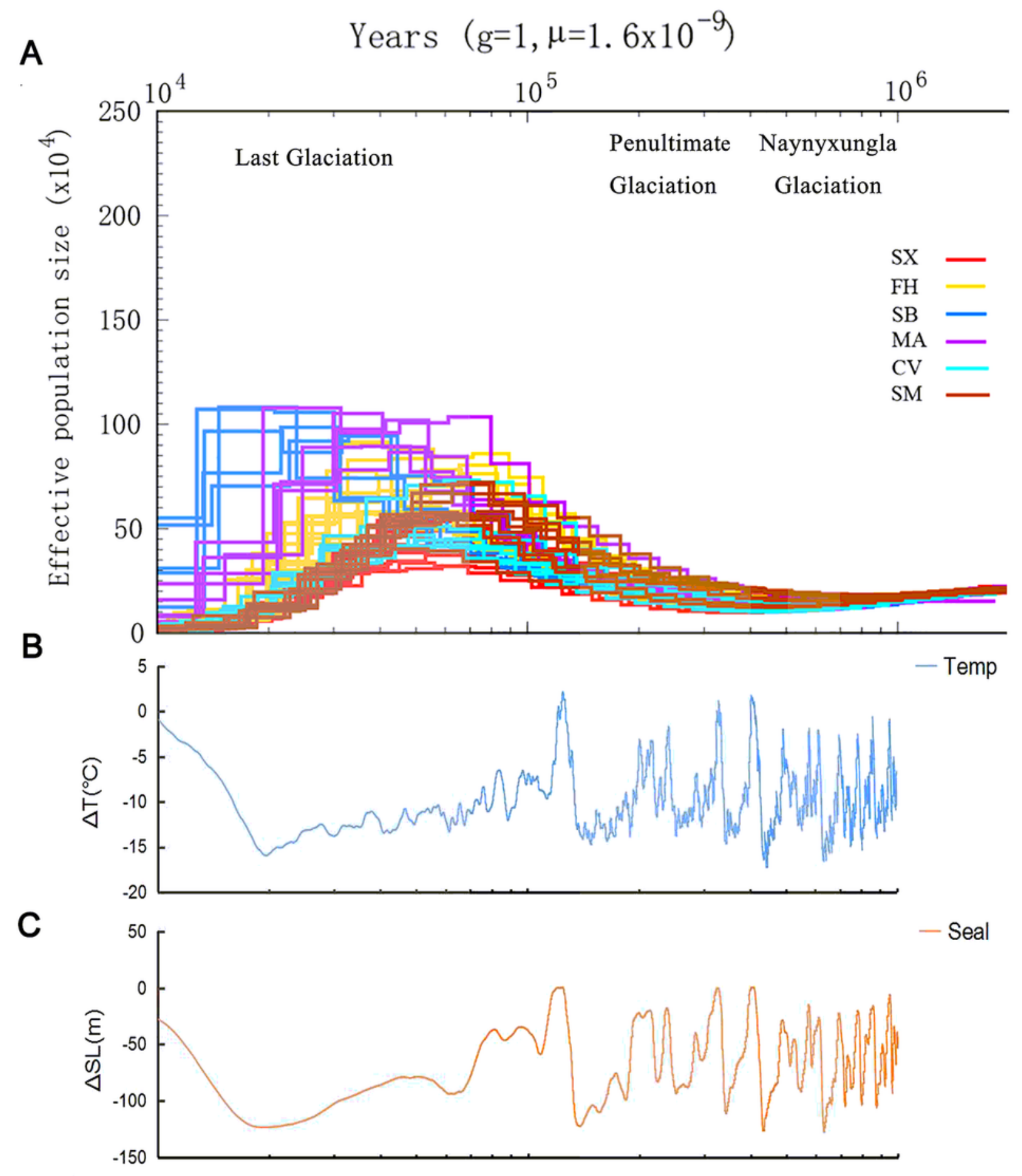

\section{Figure 3}

Demographic history of the duck populations. (A) Dynamic changes in the effective population sizes ( $\mathrm{Ne}$ ) of six duck breeds inferred by PSMC. The gray-shaded area (from left to right) refers to the Last Glaciation, the Penultimate Glaciation and the Naynyxungla Glaciation [37]. (B) The temperature from 10 KYA to 1000 KYA [38] (C) Sea level changed from 10 KYA to 1000 KYA [38]. 
A

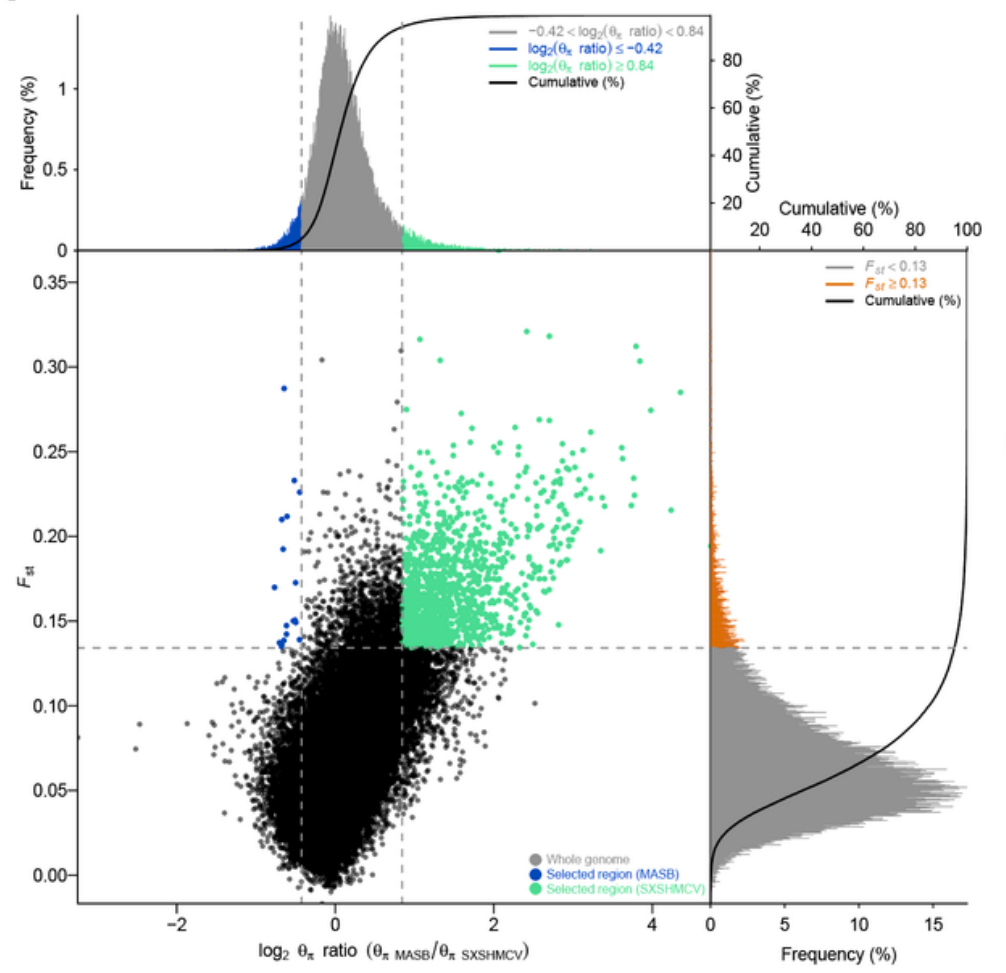

B

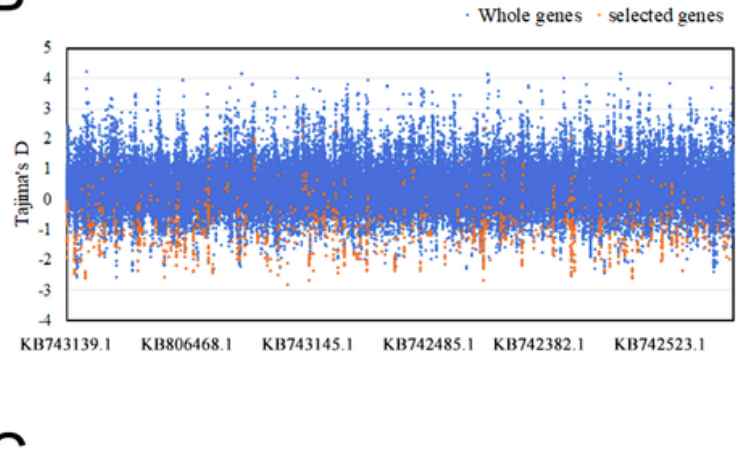

Tajima's D

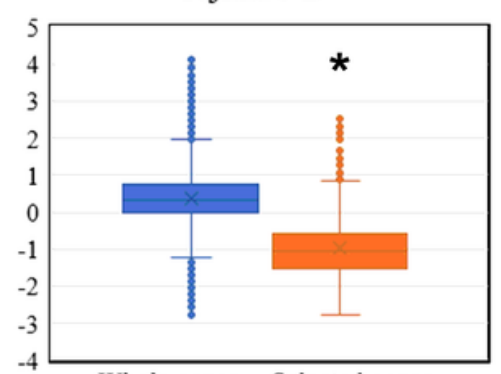

Whole genes Selected genes

\section{Figure 4}

Identification of the genomic regions with strong selective sweep signals in domestic ducks. (A) Distribution of FST values and $\log 2(\theta \pi$ ratio) calculated in $40-\mathrm{kb}$ sliding windows with $20-\mathrm{kb}$ overlap between the domestic groups and the wild groups. The data points in blue are genomic regions under selection in wild groups, and the data points in green are genomic regions under selection in the domestic groups. (B) Distribution of Tajima's D values for the whole genome and selected genes of domestic ducks. (C) Box plots of Tajima's D values for the whole genome and selected genes of domestic ducks. *Indicates a significantly elevated Tajima's $D$ relative to the whole genes (Mann-Whitney U test $P<0.05$ ). 
A

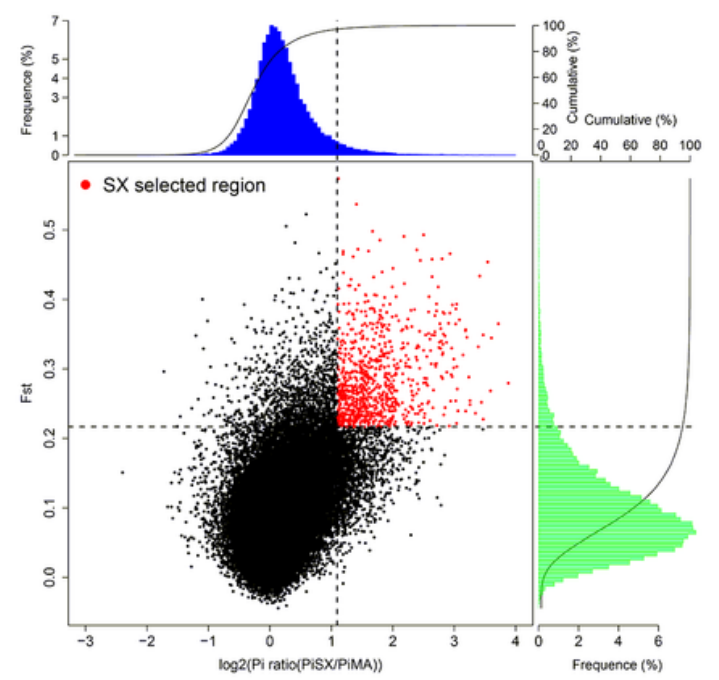

B

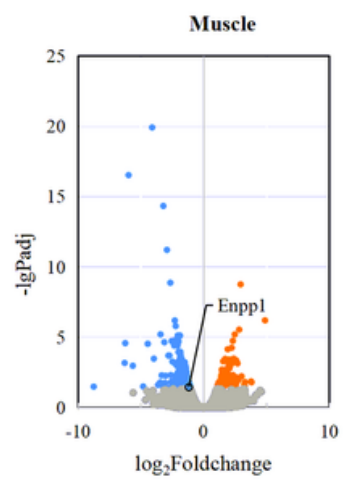

C

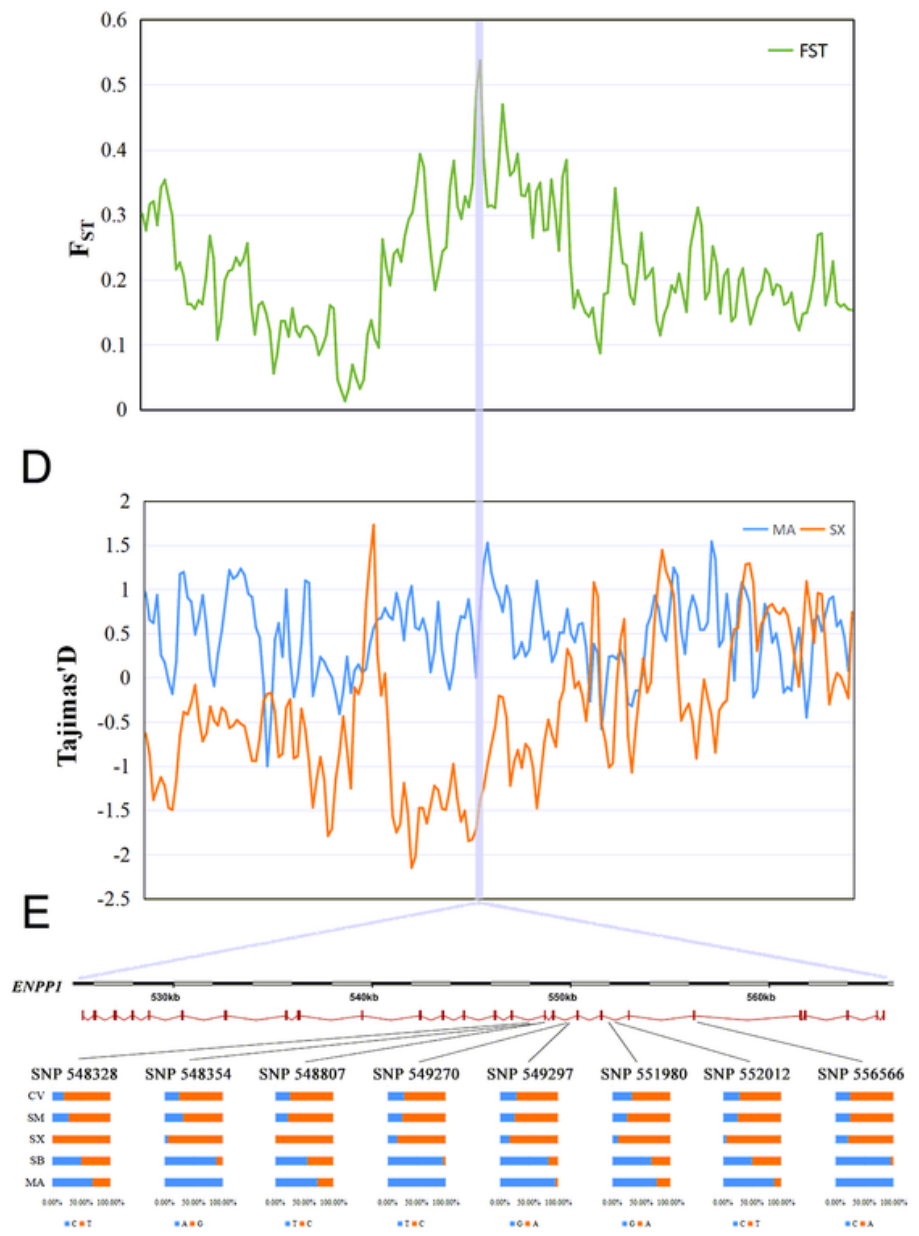

\section{Figure 5}

Genomic regions with strong selective signals in the Shaoxing duck and mallard. (A) Distribution of FST values and $\log 2(\theta \pi$ ratio) calculated in 40-kb sliding windows with $20-k b$ overlap between Shaoxing duck and mallard. The data points in red are genomic regions under selection in the SX duck. (B) Volcano plot of the differentially expressed genes in the breast muscle and liver of the Shaoxing duck and mallard. Orange splashes indicate significantly upregulated genes. Blue splashes indicate significantly downregulated genes. Grey splashes indicate genes without significant different expression. (C) FST values around the Enpp1 locus. (D) Tajima's D values around the Enpp1 locus. The blue and orange lines represent the mallard and Shaoxing duck, respectively. (E) Allele frequencies of eight SNPs within the Enpp1 gene across 6 groups. 


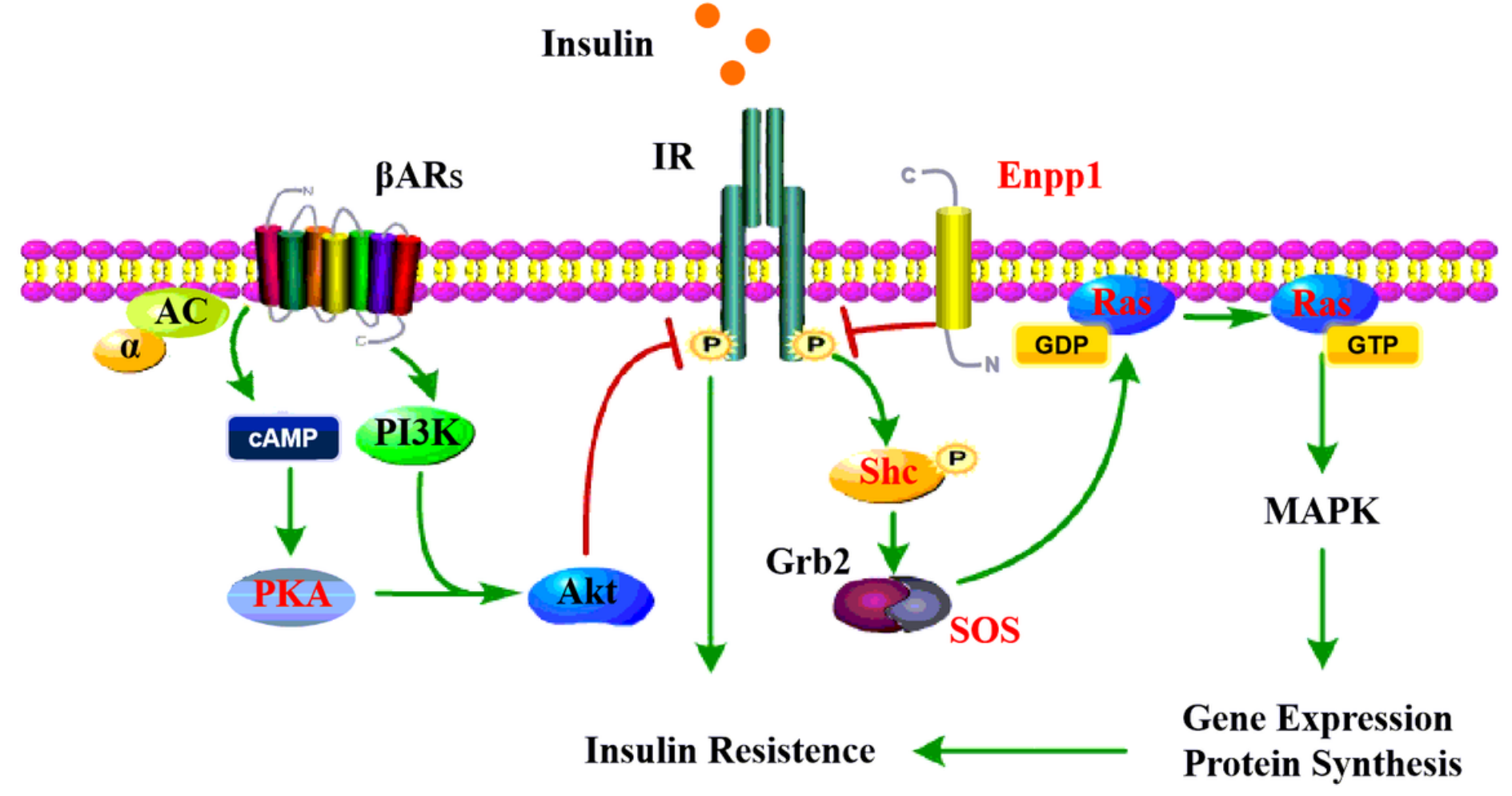

Figure 6

Selection of the insulin signaling pathway $[64,65]$. The candidate genes under selection in duck domestication are shown in red.

\section{Supplementary Files}

This is a list of supplementary files associated with this preprint. Click to download.

- Supplementary.docx 